\title{
Circulating miRNAs as predictors for morbidity and mortality in coronary artery disease
}

\author{
Barbara Mayr $^{1}\left[\right.$ D J Josef Niebauer ${ }^{1} \cdot$ Hannelore Breitenbach-Koller ${ }^{2}$
}

Received: 2 May 2019 / Accepted: 2 July 2019 / Published online: 9 July 2019

(c) The Author(s) 2019

\begin{abstract}
Micro ribonucleic acids (miRNAs) are small non-coding RNA molecules that control gene expression by translational inhibition. They have been identified to play a role in a multitude of physiological and pathophysiological cellular processes amongst others in the heart. Due to their ability to be released into the blood as well as their stability in body fluids, they appear suitable as biomarkers. This review discusses the role of selected miRNA that currently emerge as biomarkers for coronary artery disease, their potential to discriminate between different diseases, as well as how they might be used as predictive tools for cardiac events or disease outcome. Furthermore, we propose procedural steps of miRNA analysis, to allow better comparison between studies in the future.
\end{abstract}

Keywords Biomarker $\cdot$ microRNA $\cdot$ CAD

\section{Introduction}

Coronary artery disease (CAD) is the most prevalent cause of mortality worldwide [1,2]. Morbidity and mortality of CAD increases with age due to the failure to repair molecular damage, genomic instability, cellular senescence, and oxidative injuries, to name a few [3]. On a phenotypic level, the development of CAD is described by progressive erosion of the endothelium, formation of plaques and increasing stenosis of coronary arteries. The most sudden manifestation of CAD is acute myocardial infarction (AMI) [2]. Presently, in addition to electrocardiogram (ECG) evaluation, diagnosis of AMI relies on the assessment of blood based sensitive and specific biomarkers such as cardiac troponins [4]. The analysis of cardiac troponins is very accurate; nonetheless, cases of false positive or false negative results remain. Therefore, the search for specific and sensitive biomarkers

Barbara Mayr

ba.mayr@salk.at

1 University Institute of Sports Medicine, Prevention and Rehabilitation and Research Institute of Molecular Sports Medicine and Rehabilitation, Paracelsus Medical University, Salzburg, Lindhofstraße 20, 5020 Salzburg, Austria

2 Department of Bioscience, University of Salzburg, Salzburg, 5020 Salzburg, Austria is still ongoing [5]. Micro ribonucleic acid (miRNA) modulates a plethora of cellular processes throughout the complete human lifespan [6]. The endogenous, non-coding small RNAs manipulate gene expression by controlling translation of mRNAs, primarily by translational inhibition and destabilization [7]. MiRNAs have been reported to exhibit tissue-specific expression patterns during healthy states and this pattern changes during development and establishment of disease, including CAD. For diagnostic purposes, it is important to note that increase or decrease of miRNAs can be conveniently monitored from plasma or serum samples.

\section{Biogenesis of miRNAs}

MiRNA genes can be transcribed from individual miRNA genes, from introns of protein-coding genes or are polycistronic transcripts, all of which are transcribed by RNA polymerase II and are called pri-miRNA (Fig. 1, (1) [7-10]. The nuclear cleavage of the primary RNA (pri-miRNA), as the first step of the miRNA biogenesis, leads to $\sim 60-70 \mathrm{nt}$ stem loop intermediates called pre-miRNA. This cleavage is performed by the RNase III endonuclease enzyme Drosha, which cleaves both strands near the base of the primary stem loop (Fig. 1, (2)). Those short RNA hairpins bearing a 2-nt $3^{\prime}$ overhang, as well as a $5^{\prime}$ phosphate are then bound by the nuclear export factor Ran-GTP/Exportin 5, which 
Fig. 1 Schema of miRNA maturation. (1) Polymerase II transcribe miRNA gene into pri-miRNA; (2) Drosha cleave pri-miRNA into pre-miRNA; (3) RNA-GTP/Exportin5 transport pre-miRNA into the cytoplasm; (4) Dicer cleave pre-miRNA into miRNA:miRNA* duplex; (5) Helicase split up duplex into mature miRNAs; (6) miRNA are loaded onto the RISC; (7) RISC:miRNA complex work through translational repression; (8) or through mRNA degradation; $O R F$ open reading frame, RISC RNA-induced silencing complex

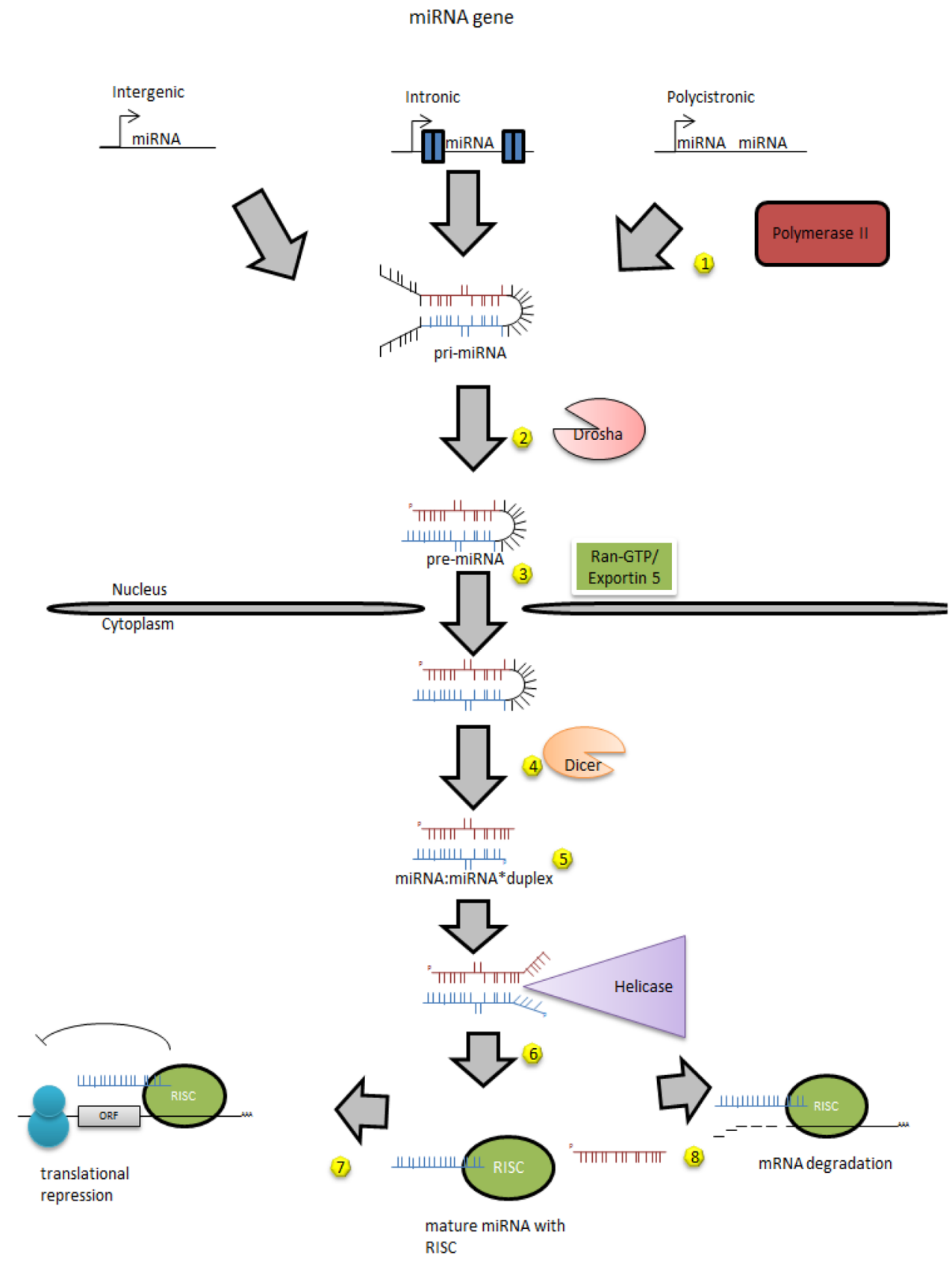

is responsible for the transport of the pre-miRNA into the cytoplasm (Fig. 1, (3) [8-10]. The maturation of miRNAs occurs in the cytoplasm, where the second RNase III endonuclease enzyme Dicer recognizes the double-stranded portion of the pre-miRNA. Dicer cuts both strands about two helical turns away from base of the stem loop, generating a duplex composed of two similar sized fragments, one with a $5^{\prime}$ phosphate and one with a 2-nt 3 ' extension of the miRNA. These fragments are called miRNA*-sequences and form a double-stranded miRNA:miRNA* duplex (Fig. 1, (4) $[9,10]$. A helicase splits the double-stranded duplex into two single stranded miRNAs, which then form the mature miRNAs, often with preferential use of one of the miRNAs (Fig. 1, (5). The mature miRNAs are loaded into RNA-induced silencing complex (RISC) [11], where they function as a navigation system for RISC to dock unto the complementary mRNA target (Fig. 1, (6). When bound to a specific mRNA the RISC:miRNA complex functions either through translational repression (Fig. 1, (7) ) or through mRNA degradation (Fig. 1, 8) [8-10]. 


\section{miRNAs as biomarker}

Biomarkers are ideally ubiquitously present in easy collectable such as in blood. Also, they need to strong predictability of detection and robust reliability, i.e. excellent specificity and sensitivity. Circulating miRNAs appear to be suitable candidates as biomarkers since they are easily accessible, and are known for their stability and specificity in plasma or serum [12]. The miRNAs which are most abundant in the skeletal or heart muscle, are the socalled myomiRs. Of these, miR-1, miR-133, miR-208 and miR-499 are among the most intensively characterized miRNAs in investigations of cardiac pathologies. All four miRNAs have increased expression levels in plasma/serum samples of AMI patients. MiR-208 is solely expressed in cardiomyocytes and it is released into the blood stream during cardiac cell death in AMI, whereas it is mostly undetectable in the circulation of healthy individuals. The miR-208 expression pattern after an AMI is similar to the cardiac specific troponins [13]. In a set of patients with AMI, Wang et al. described an increase in serum levels of miR-208a within $4 \mathrm{~h}$ after onset of symptoms, whereas only $85 \%$ of these patients had elevated troponin levels. All four myomiRs were expressed at a higher level in AMI patients when compared to non-AMI patients [14].

Some miRNAs, like the miR-499-5p, have also been described to have diagnostic values. They allow discriminating between patients with non-ST elevated myocardial infarction (NSTEMI) not only from controls, but also from patients with acute heart failure without AMI. Thus, in this subset of cardiac patients, miR-499-5p was superior to cardiac troponins, both with respect to the area of application as well as discriminatory power [15]. Moreover, miRNAs have been associated with the presence of CAD, i.e. miR-133a, miR-134, miR-145, miR-122 and miR370 , have been found to correlate with severity of disease [16-18]. A question that remains is whether miRNAs might be able to discriminate between patients with stable (SA) versus unstable angina (UA) [19]. Likewise, it is of interest whether miRNAs could be used to discriminate between patients with stable angina pectoris and those with acute myocardial infarction. Widera et al. showed that miR-1, miR-133a and miR-208b had significantly higher expression levels in NSTEMI or ST elevated myocardial infarctions (STEMI) than in UA, whereas there was no difference in miR-133b, miR-208a and miR-499. However, each of the miRNAs showed expression overlaps between the examined diseases [20]. In addition to myomiRs, other miRNAs have been reported which are able to discriminate between the different forms of cardiac disease. Ren and colleagues found that miR-21, miR-25, miR-92, miR-106b, miR-126 and miR-451 were significantly elevated in UA compared to SA and healthy controls [21]. Another study reported that miR-186, miR-132 and miR-150 are differentially expressed in patients with UA, patients with chest pain of non-cardiac origin and healthy controls, respectively [22].

\section{miRNAs as predictor for future personal risk status}

One of the most common risk scores for the prediction of cardiovascular diseases (CVD) is the Framingham Risk Score (FRS), which includes gender, age, cholesterol level, high-density lipoprotein level as well as systolic blood pressure [23]. Most recently, miRNA expression levels have been added to this and other scores to improve predictability of CVD [24].

Bye and colleagues postulated a panel of five miRNAs, which revealed an enhanced risk prediction of suffering a fatal AMI in putatively healthy individuals in a 10-years observation period. With this panel $78 \%$ of the observed cases were correctly classified. These authors suggested using the miRNA expression levels of miR-106a-5p, miR424-5p, let-7 g-5p, miR-144-3p and miR-660-5p, which improved the predictive power of FRS even further, i.e. increased the area under the curve from 0.72 to 0.91 [24].

Conversely, we have previously published a tool for discrimination between healthy subjects and patients with CAD, which may even be used for identification of subjects at risk for exercise-induced cardiac events. Using miRNA resting expression levels of miR-150-5p as well as the modulated expression levels after a maximal ergometry of miR101-3p, miR-141-3p and miR-200b-3p, 70\% of the cases were correctly classified as patients with CAD. Including the performance parameters of maximal oxygen uptake as well as maximum exercise capacity corrected for the bodyweight the correct classification of CAD cases was raised to $92.5 \%$ [25].

\section{Challenges in miRNA study comparisons}

The ever-increasing number of studies aiming to identify new biomarkers for specific conditions mirrors the interest in miRNAs as prognostic markers in disease. There are several aspects, both methodological as well as biological ones, which have to be considered before assigning high prognostic potential to a given miRNA. First, consideration has to be given to availability and accessibility of sample material for analysis, e.g. whole blood, plasma, serum, different blood cell lines, and saliva. Second, there is also a great variety of methods used for miRNA analysis. Not only are there different methods for the identification and 
quantification of miRNAs, there exists also a high number of different procedures for pre-analytic assessment of samples as well as of isolation methods. Third, there is no universal normalization methods. Early biomarker studies normalized their miRNAs against certain miRNAs like miR-16. Unfortunately, this miRNA has later been identified as one of many miRNAs that are highly expressed in red blood cells. Consequently, detection levels are highly influenced by the degree of hemolysis of the samples [26]. In addition, results of miRNA analysis, amongst others also of miR16 , are dependent on the handling of the samples itself, as centrifugation or filtration steps prior to the isolation of the RNA lead to variations of the outcome [27]. Indeed, we have previously shown that a single filtration step for plasma samples prior to the isolation of miRNAs improve the variability of measurements significantly [27].

Additional aspects like gender have to be taken into account [28]. Also, the miRNA expression profile changes throughout the aging process [28] and miRNA expression levels are strongly influenced by acute exercise [25], but also by sustained exercise [29].

\section{Conclusion}

MiRNAs have the potential to serve as biomarkers for CAD and CAD related diseases. Current reports provide a large number of miRNAs as possible candidates. However, most human studies used diverse preparation protocols and rather small sample sizes. In addition, medication like statins and other are not always sufficiently accounted for.

We conclude that further studies have to be conducted in rather homogeneous study populations, have to enroll more patients and ought to adhere to universally used and therefore comparable preparation methods. Thus, at present the prognostic potential of single miRNAs is not yet superior to well-established biomarkers exemplified by cardiac troponins. However, combinatorial use of the latter with miRNAs might improve predictability even further.

If procedural recommendations are followed, miRNAs might emerge as biomarkers superior to those currently used.

Acknowledgements Open access funding provided by Paracelsus Medical University.

Open Access This article is distributed under the terms of the Creative Commons Attribution 4.0 International License (http://creativeco mmons.org/licenses/by/4.0/), which permits unrestricted use, distribution, and reproduction in any medium, provided you give appropriate credit to the original author(s) and the source, provide a link to the Creative Commons license, and indicate if changes were made.

\section{References}

1. GBD 2013 Mortality and Causes of Death Collaborators (2015) Global, regional, and national age-sex specific all-cause and cause-specific mortality for 240 causes of death, 1990-2013: a systematic analysis for the Global Burden of Disease Study 2013. Lancet 385(9963):117-171

2. Benjamin EJ, Blaha MJ, Chiuve SE et al (2017) Heart disease and stroke statistics-2017 update: a report from the American Heart Association. Circulation 135(10):e146-e603

3. Bronze-da-Rocha E (2014) MicroRNAs expression profiles in cardiovascular diseases. Biomed Res Int 2014:985408

4. Thygesen K, Alpert JS, Jaffe AS et al (2012) Third universal definition of myocardial infarction. J Am Coll Cardiol 60(16):1581-1598

5. Jaffe AS, Ravkilde J, Roberts R et al (2000) It's time for a change to a troponin standard. Circulation 102(11):1216-1220

6. Ambros V (2004) The functions of animal microRNAs. Nature 431(7006):350-355

7. Kloosterman WP, Plasterk RH (2006) The diverse functions of microRNAs in animal development and disease. Dev Cell 11(4):441-450

8. Cai X, Hagedorn CH, Cullen BR (2004) Human microRNAs are processed from capped, polyadenylated transcripts that can also function as mRNAs. RNA 10(12):1957-1966

9. van Rooij E, Olson EN (2007) MicroRNAs: powerful new regulators of heart disease and provocative therapeutic targets. J Clin Invest 117(9):2369-2376

10. Bartel DP (2004) MicroRNAs: genomics, biogenesis, mechanism, and function. Cell 116(2):281-297

11. Maron BJ, Thompson PD, Puffer JC et al (1998) Cardiovascular preparticipation screening of competitive athletes: addendum: an addendum to a statement for health professionals from the Sudden Death Committee (Council on Clinical Cardiology) and the Congenital Cardiac Defects Committee (Council on Cardiovascular Disease in the Young), American Heart Association. Circulation 97(22):2294

12. Reid G, Kirschner MB, van Zandwijk N (2011) Circulating microRNAs: association with disease and potential use as biomarkers. Crit Rev Oncol Hematol 80(2):193-208

13. Schulte C, Karakas M, Zeller T (2017) microRNAs in cardiovascular disease-clinical application. Clin Chem Lab Med 55(5):687-704

14. Wang GK, Zhu JQ, Zhang JT et al (2010) Circulating microRNA: a novel potential biomarker for early diagnosis of acute myocardial infarction in humans. Eur Heart J 31(6):659-666

15. Olivieri F, Antonicelli R, Lorenzi M et al (2013) Diagnostic potential of circulating miR-499-5p in elderly patients with acute non ST-elevation myocardial infarction. Int $\mathrm{J}$ Cardiol 167(2):531-536

16. Gao W, He HW, Wang ZM et al (2012) Plasma levels of lipometabolism-related miR-122 and miR-370 are increased in patients with hyperlipidemia and associated with coronary artery disease. Lipids Health Dis 15(11):55

17. Gao H, Guddeti RR, Matsuzawa Y et al (2015) Plasma levels of microRNA-145 are associated with severity of coronary artery disease. PLoS ONE 10(5):e0123477

18. Wang F, Long G, Zhao C et al (2013) Plasma microRNA-133a is a new marker for both acute myocardial infarction and underlying coronary artery stenosis. J Transl Med 23(11):222

19. Chen MC, Chang TH, Chang JP et al (2016) Circulating miR$148 b-3 p$ and miR-409-3p as biomarkers for heart failure in patients with mitral regurgitation. Int J Cardiol 01(222):148-154 
20. Widera C, Gupta SK, Lorenzen JM et al (2011) Diagnostic and prognostic impact of six circulating microRNAs in acute coronary syndrome. J Mol Cell Cardiol 51(5):872-875

21. Ren J, Zhang J, Xu N et al (2013) Signature of circulating microRNAs as potential biomarkers in vulnerable coronary artery disease. PLoS ONE 8(12):e80738

22. Zeller T, Keller T, Ojeda F et al (2014) Assessment of microRNAs in patients with unstable angina pectoris. Eur Heart $\mathrm{J}$ 35(31):2106-2114

23. D'Agostino RB Sr, Vasan RS, Pencina MJ et al (2008) General cardiovascular risk profile for use in primary care: the Framingham Heart Study. Circulation 117(6):743-753

24. Bye A, Rosjo H, Nauman J et al (2016) Circulating microRNAs predict future fatal myocardial infarction in healthy individualsthe HUNT study. J Mol Cell Cardiol 97:162-168

25. Mayr B, Mueller EE, Schäfer C et al (2019) Exercise responsive micro ribonucleic acids identify patients with coronary artery disease. Eur J Prev Cardiol 26(4):348-355

26. Kirschner MB, Edelman JJ, Kao SC, Vallely MP, van Zandwijk N, Reid G (2013) The impact of hemolysis on cell-free microRNA biomarkers. Front Genet 4:94
27. Mayr B, Mueller EE, Schäfer C, Breitenbach-Koller H, Schönfelder M, Niebauer J (2017) Pitfalls of analysis of circulating miRNA: role of hematocrit. Clin Chem Lab Med 55(5):622-625

28. Ameling S, Kacprowski T, Chilukoti RK et al (2015) Associations of circulating plasma microRNAs with age, body mass index and sex in a population-based study. BMC Med Genom 14(8):61

29. Baggish AL, Hale A, Weiner RB et al (2011) Dynamic regulation of circulating microRNA during acute exhaustive exercise and sustained aerobic exercise training. J Physiol 589(Pt 16):3983-3994

Publisher's Note Springer Nature remains neutral with regard to jurisdictional claims in published maps and institutional affiliations. 